PROCEEDINGS OF THE

AMERICAN MATHEMATICAL SOCIETY

Volume 129, Number 12, Pages 3511-3518

S 0002-9939(01)06290-6

Article electronically published on July 17, 2001

\title{
MAXIMAL POINCARÉ POLYNOMIALS AND MINIMAL MORSE FUNCTIONS
}

\author{
V. BENCI AND K. A. DE REZENDE
}

(Communicated by Michael Handel)

\begin{abstract}
In this paper we introduce the maximum Poincaré polynomial $P^{*}(M)$ of a compact manifold $M$, and prove its uniqueness. We show that its coefficients are topological invariants of the manifolds which, in some cases, correspond to known ones. We also investigate its realizability via a Morse function on $M$.
\end{abstract}

\section{INTRODUCTION}

The set of Poincaré polynomials of a manifold $M, \mathcal{P}(M)$, has the property that the coefficients of polynomials in this set are topological invariants of $M$. In this paper, we prove that there exists a unique maximal Poincaré polynomial, $P^{*}$. In some sense, we are choosing a polynomial in $\mathcal{P}(M)$ which carries the greatest amount of topological information about $M$.

We also study the realizability of $P^{*}$ as a Morse function of $M$, which we refer to as a minimal Morse function. This brings up interesting questions as to the continuation and bifurcation of flows on $M$. Consider the Morse relations for flows with Morse functions which are associated to a realizable maximal Poincaré polynomial $P^{*}$. The remainder term in this Morse relation represents in some sense extra critical points or critical sets that can either collapse, bifurcate or cancel among themselves. We do not know if this is always the case. It is possible that if $P^{*}$ is not realizable, then the remainder terms may be topological invariants. In any case, these are questions that remain unanswered.

\section{PoincarÉ POLYNOMIALS}

Let $M$ be a differentiable $n$-manifold. Let $\chi(M)$ be the Euler characteristic of $M$ and $\mathcal{F}(M)$ the set of Morse functions on $M$.

Let $\mathcal{S}$ denote the set of polynomials in the variable $\lambda$ with integer coefficients $c_{k}$. Let $P(\lambda), Q(\lambda) \in \mathcal{S}$ where $P(\lambda)=\sum c_{k}(P) \lambda^{k}$ and $Q(\lambda)=\sum c_{k}(Q) \lambda^{k}$. Define the minimum of $P$ and $Q$ to be $P \wedge Q=\sum \min \left\{c_{k}(P), c_{k}(Q)\right\} \lambda^{k}$. The operation $\wedge$ gives the usual partial order relation defined as follows: $P \leq Q: \leftrightarrow P \wedge Q=P$.

Received by the editors December 7, 1999.

2000 Mathematics Subject Classification. Primary 37D15, 37C10; Secondary 54H20, 37B30.

This research was supported by the Conselho Nacional de Desenvolvimento Científico e Tecnológico under Grant 300072/90.2. 
Define the maximum of $P$ and $Q$ to be $P \vee Q=\sum \max \left\{c_{k}(P), c_{k}(Q)\right\} \lambda^{k}$. The operation $\vee$ gives the usual partial order relation defined as follows: $P \geq Q: \leftrightarrow$ $P \vee Q=P$.

Note that the operation $\wedge$ (resp. $\vee$ ) makes sense for an infinite family of polynomials $\left\{P_{i}\right\}_{i \in I}$ if the coefficients are bounded from below (resp. bounded from above). In this case we have $\bigwedge_{i \in I} P_{i}=\sum\left\{\min _{i \in I} c_{k}\left(P_{i}\right)\right\} \lambda^{k}$. Similarly for the $\vee$ operation.

Denote by $m_{f}(\lambda)$ the Morse polynomial associated to the Morse function $f$ with coefficients $c_{k}(f)$. Let $\mathcal{B}(M)$ denote the set of Betti polynomials on $M$, that is, the subset of $\mathcal{S}$ consisting of those polynomials $P$ whose coefficients $c_{k}(P)$ are the Betti numbers of $M$, taken with respect to some field coefficient $F$, i.e., $c_{k}(P)=\beta_{k}(M ; F)$. These are also referred to in the literature as Poincaré polynomials. However, throughout this paper we will refer to these polynomials as Betti polynomials and reserve the term Poincaré polynomials to mean generalized Poincaré polynomials as defined subsequently.

Definition 2.1. The set of generalized Poincaré polynomials on $M$ is defined by the following properties:

$P \in \mathcal{P}(M)$ if and only if

(1) $P \geq \mathbf{0}$, i.e., $c_{k}(P) \geq 0 \quad \forall k$;

(2) $\forall f \in \mathcal{F}(M) \quad \exists Q_{f} \geq \mathbf{0}$ such that $m_{f}(\lambda)=P(\lambda)+(1+\lambda) Q_{f}(\lambda)$.

We will refer to these generalized Poincaré polynomials, in short, as Poincaré polynomials. In dimensions greater than two, $\mathcal{P}(M)$ may contain more than one element. For instance, if $n$ is odd we always have that $\lambda^{n}+1 \in \mathcal{P}(M)$. If $n$ is even, $\lambda^{n}+k \lambda^{\frac{n}{2}}+1 \in \mathcal{P}(M)$. In both of the previous cases these need not be $P^{*}$.

Clearly, $\mathcal{B}(M) \neq \emptyset$, and by Morse theory it follows that any Betti polynomial is a Poincaré polynomial.

The next theorem will establish properties satisfied by (generalized) Poincaré polynomials.

Theorem 2.2. (1) $P(-1)=\chi(M) \quad \forall P \in \mathcal{P}(M)$;

(2) $c_{k}(f) \geq c_{k}(P) \quad \forall f \in \mathcal{F}(M)$ and $\forall P \in \mathcal{P}(M)$ (generalized weak Morse inequalities);

(3) $c_{k}(f)-c_{k-1}(f)+\ldots \pm c_{0}(f) \geq c_{k}(P)-c_{k-1}(P)+\ldots \pm c_{0}(P), \forall k, \forall f \in \mathcal{F}(M)$ and $\forall P \in \mathcal{P}(M)$ (generalized Morse inequalities).

Proof. Clearly if we take a Betti polynomial $B \in \mathcal{B}(M): m_{f}(-1)=B(-1)=$ $\chi(M)$. On the other hand, given $P \in \mathcal{P}(M), m_{f}(\lambda)=P(\lambda)+(1+\lambda) Q_{f}(\lambda)$, hence $m_{f}(-1)=P(-1)$, proving $(1)$.

The second statement also follows trivially, since $\forall f \in \mathcal{F}(M)$ and $\forall P \in \mathcal{P}(M)$ we have that $m_{f}(\lambda)=P(\lambda)+(1+\lambda) Q_{f, P}(\lambda)$, where $Q_{f, P} \geq 0$. Hence $c_{k}(f) \geq c_{k}(P)$.

For the third statement, we have that:

$$
\sum_{i=0}^{n} c_{i}(f) \lambda^{i}=\sum_{i=0}^{n} c_{i}(P) \lambda^{i}+(1+\lambda) \sum_{i=0}^{n-1} c_{i}(Q) \lambda^{i}
$$

where $n$ is the dimension of $M$. When this expression is calculated at $\lambda=-1$ we obtain the top Morse inequality (which is actually an equality). If we sum up to $k<n, \sum_{i=0}^{k} c_{i}(f) \lambda^{i}=\sum_{i=0}^{k} c_{i}(P) \lambda^{i}+c_{k}(Q) \lambda^{k}+\sum_{i=0}^{k-1} c_{i}(Q)\left(\lambda^{i}+\lambda^{i+1}\right)$. Therefore, $\sum_{i=0}^{k}(-1)^{i} c_{i}(f)=\sum_{i=0}^{k}(-1)^{i} c_{i}(P)+(-1)^{k} c_{k}(Q)$. If $k$ is odd, multiply both sides 
of the above equality by -1 , and if $k$ is even the equality remains as it is. In both cases we obtain $\sum_{i=0}^{k}(-1)^{i} c_{i}(f) \geq \sum_{i=0}^{k}(-1)^{i} c_{i}(P)$ since $c_{k}(Q) \geq 0$.

2.1. Maximal Poincaré polynomials. It is interesting to note that in defining an element $P \in \mathcal{P}(M)$ we are in some sense minimizing over all possible polynomials in $\mathcal{S}$ and considering those which have the property that every Morse function on $M$ has at least $c_{k}(P)$ critical points of index $k$.

It is then a natural consideration to attempt to define a maximum polynomial in this family $\mathcal{P}(M)$. The question at this point is the following: does such a maximum exist and if so how can we define it? There are several natural ways in which one can define a notion of maximality in this family.

The first way uses the lattice relation, that is, $\max _{P \in \mathcal{P}(M)} P=\bar{P}$ if and only if $\bar{P} \geq P$ for all $P \in \mathcal{P}(M)$. In this case, it follows that $c_{k}(\bar{P}) \geq c_{k}(P)$ for each $k$. We remark that if this maximum $\bar{P}$ exists it is unique by definition. Also $\forall f \in \mathcal{F}(M)$ the coefficients of the associated Morse polynomial $c_{k}(f)$ satisfy $c_{k}(f) \geq c_{k}(\bar{P})$. We refer to $\bar{P}$ as the maximum Poincaré polynomial.

A second possible way in which a notion of maximality may be defined is to let $b_{1}(M)=\max _{P \in \mathcal{P}(M)} P(1)$ and to consider the subset $\Sigma_{1}(M)=\{P \in \mathcal{P}(M)$ : $\left.P(1)=b_{1}(M)\right\}$. We refer to $\Sigma_{1}$ as the set of maximum Poincaré polynomials. In this way one captures among the Poincaré polynomials those which maximize the total sum of the coefficients, that is, those $P \in \mathcal{P}(M)$ such that $\sum c_{k}(P)=b_{1}(M)$.

The following proposition will show that if $\bar{P}$ exists, $\Sigma_{1}(M)$ is a singleton, i.e., $\Sigma_{1}(M)=\{\bar{P}\}$.

Proposition 2.3. If $\bar{P}$ exists, then $\Sigma_{1}(M)=\{\bar{P}\}$.

Proof. If $\bar{P}$ exists, then $b_{1}(M)=\sum c_{k}(\bar{P})$. Also, if $P^{\prime} \in \Sigma_{1}$ and $P^{\prime} \neq \bar{P}$, then $\forall k, 0 \leq c_{k}\left(P^{\prime}\right) \leq c_{k}(\bar{P})$ and for some $j, c_{j}\left(P^{\prime}\right)<c_{j}(\bar{P})$. Moreover, since $P^{\prime} \in \Sigma_{1}$, $b_{1}(M)=\sum c_{k}\left(P^{\prime}\right)$. However, since $c_{j}\left(P^{\prime}\right)<c_{j}(\bar{P})$, we have:

$$
\sum c_{k}\left(P^{\prime}\right)<\sum c_{k}(\bar{P})
$$

a contradiction.

However, even if $\bar{P}$ does not exist we will prove that $\Sigma_{1}(M)$ is a singleton.

Proposition 2.4. Every $P \in \Sigma_{1}$ satisfies the following properties:

(1) $P(-1)=\chi(M)$.

(2) $\forall f \in \mathcal{F}(M), c_{k}\left(m_{f}\right) \geq c_{k}(P)$.

(3) $\forall k, \exists f \in \mathcal{F}(M), c_{k}\left(Q_{f}\right)=0$.

Proof. (1) and (2) follow from Theorem 2.2; let us prove (3). Suppose that (3) is false; then

$$
\exists k, \forall f \in \mathcal{F}(M), c_{k}\left(Q_{f}\right)>0 .
$$

Then the following polynomial is a Poincaré polynomial:

$$
P_{1}(\lambda)=P(\lambda)+(1+\lambda) \lambda^{k} .
$$

This follows from the fact that, $\forall f \in \mathcal{F}(M)$,

$$
m_{f}(\lambda)=P(\lambda)+(1+\lambda) \lambda^{k}+(1+\lambda)\left(Q_{f}(\lambda)-\lambda^{k}\right)=P_{1}(\lambda)+(1+\lambda) Q_{1}(\lambda),
$$

where $Q_{1}(\lambda)=Q_{f}(\lambda)-\lambda^{k}$. We write $Q_{f}(\lambda)$ in short for $Q_{f, P}(\lambda)$ and $Q_{1}(\lambda)$ in short for $Q_{f, P_{1}}(\lambda)$. Since $Q_{1}(\lambda)=Q_{f}(\lambda)-\lambda^{k}=\left(\sum c_{k}\left(Q_{f}\right) \lambda^{k}\right)-\lambda^{k}$ we conclude 
that $c_{j}\left(Q_{1}\right)=c_{j}\left(Q_{f}\right)>0$ for $j \neq k$ and $c_{k}\left(Q_{1}\right)=c_{k}\left(Q_{f}\right)-1$ which is also greater than or equal to zero because of our hypothesis $c_{k}\left(Q_{f}\right)>0$. Hence, $P_{1}(\lambda)$ is a Poincaré polynomial.

Now, $P_{1}(1)=P(1)+2=b_{1}(M)+2$ which contradicts the maximality of $b_{1}(M)$.

Theorem 2.5. $\Sigma_{1}(M)$ is a singleton.

Proof. Let $P_{1}$ and $P_{2}$ be two different maximal polynomials, and let $k$ be the minimum integer such that $c_{k}\left(P_{1}\right) \neq c_{k}\left(P_{2}\right)$. Without loss of generality we may suppose that $c_{k}\left(P_{1}\right)<c_{k}\left(P_{2}\right)$. Now by Proposition 2.3 let $f \in \mathcal{F}(M)$ be a function such that

$$
m_{f}(\lambda)=P_{1}(\lambda)+(1+\lambda) Q_{1}(\lambda)
$$

with $c_{k}\left(Q_{1}\right)=0$ where we write $Q_{1}(\lambda)$ in short for $Q_{f, P_{1}}(\lambda)$. Also, the following equation holds for $m_{f}(\lambda)$ :

$$
m_{f}(\lambda)=P_{2}(\lambda)+(1+\lambda) Q_{2}(\lambda),
$$

where we write $Q_{2}(\lambda)$ in short for $Q_{f, P_{2}}(\lambda)$.

For $0<m \leq k$ the two equations above imply the following relations among the coefficients of the polynomials involved:

$$
\left\{\begin{array}{l}
c_{k-m}\left(m_{f}\right)=c_{k-m}\left(P_{2}\right)+c_{k-m}\left(Q_{2}\right)+c_{k-m-1}\left(Q_{2}\right), \\
c_{k-m}\left(m_{f}\right)=c_{k-m}\left(P_{1}\right)+c_{k-m}\left(Q_{1}\right)+c_{k-m-1}\left(Q_{1}\right) .
\end{array}\right.
$$

Note that (1) is true for $m=0$ where $c_{k}\left(Q_{1}\right)=0$.

We make the following assertion:

$$
c_{k-s}\left(Q_{2}\right)=c_{k-s}\left(Q_{1}\right)+(-1)^{s} \sigma,
$$

where $\sigma=c_{k}\left(P_{2}\right)-c_{k}\left(P_{1}\right)+c_{k}\left(Q_{2}\right)$. We assert that $\sigma>0$ and this follows from our initial assumption that $c_{k}\left(P_{1}\right)<c_{k}\left(P_{2}\right)$. Equation (2) can be proved by induction. For $s=1$ write (1) with $m=0$. Recall that $c_{k}\left(Q_{1}\right)=0$, hence (1) can be written as follows:

$$
\left\{\begin{array}{l}
c_{k}\left(m_{f}\right)=c_{k}\left(P_{2}\right)+c_{k}\left(Q_{2}\right)+c_{k-1}\left(Q_{2}\right), \\
c_{k}\left(m_{f}\right)=c_{k}\left(P_{1}\right)+c_{k-1}\left(Q_{1}\right) .
\end{array}\right.
$$

By (3) equation (2) follows for $s=1$. We assume (2) to be true for $s$ and we will prove it true for $s+1$. For this purpose, we rewrite (1) using our induction hypothesis, i.e., using (2) with $s$ and using the fact that for $1 \leq s \leq k, c_{k-s}\left(P_{1}\right)=$ $c_{k-s}\left(P_{2}\right)$.

$$
\left\{\begin{array}{l}
c_{k-s}\left(m_{f}\right)=c_{k-s}\left(P_{1}\right)+c_{k-s}\left(Q_{1}\right)+(-1)^{s} \sigma+c_{k-s-1}\left(Q_{2}\right) \\
c_{k-s}\left(m_{f}\right)=c_{k-s}\left(P_{1}\right)+c_{k-s}\left(Q_{1}\right)+c_{k-s-1}\left(Q_{1}\right)
\end{array}\right.
$$

By comparing these two equations we have that

$$
c_{k-s-1}\left(Q_{2}\right)+(-1)^{s} \sigma=c_{k-s-1}\left(Q_{1}\right)
$$

from which our assertion follows for $s+1$ as long as $1 \leq s \leq k$. At $s=k$ (2) becomes $c_{-1}\left(Q_{2}\right)=c_{-1}\left(Q_{1}\right)+(-1)^{k} \sigma$. However, this is absurd since $c_{-1}\left(Q_{2}\right)=$ $c_{-1}\left(Q_{1}\right)=0$.

The only polynomial in $\Sigma_{1}(M)$ is called the maximum Poincaré polynomial and we will denote it by $P^{*}$ and its coefficients are $\beta_{k}\left(P^{*}\right)$. 


\section{Realizability and minimal Morse functions}

We will say that a Poincaré polynomial $P$ is realizable if there exists a Morse function $f$ such that the coefficient $c_{k}(f)$ of the associated Morse polynomial of $f$ is equal to $c_{k}(P)$. If the maximal Poincaré polynomial $P^{*}$ is realizable as a Morse function $f$ on $M$ we refer to $f$ as a minimal Morse function. On the other hand, if $P^{*}$ is not realizable then there is no minimal Morse functions (in the sense above). In this case, the remainder terms, i.e., the coefficients $c_{k}(Q)$, will represent the different yet equivalent choices of pairs of critical points that are necessary to add to $P^{*}$ to make it realizable.

A theorem of Smale's implies the following realizability result [Fr].

Theorem 3.1. Let $M$ be simply connected and of dimension $>5$. If the nonnegative integers $\beta_{k}\left(P^{*}\right)$ satisfy

$$
\beta_{k}\left(P^{*}\right)-\beta_{k-1}\left(P^{*}\right)+\cdots \pm \beta_{0}\left(P^{*}\right) \geq \beta_{k}(M, F)-\beta_{k-1}(M, F)+\cdots \pm \beta_{0}(M, F)
$$

for every field $F$, then $P^{*}$ is realizable.

$P^{*}$ is in some sense a common core for all Morse functions on $M$. Hence it follows easily that the weak Morse inequalities hold for $P^{*}$.

Proposition 3.2. The weak Morse inequalities hold for $P^{*}$, i.e., $\beta_{k}\left(P^{*}\right) \geq$ $\beta_{k}(M, F)$.

Proof. By the definition of $P^{*}$, all Morse functions on $M$ have at least $\beta_{k}\left(P^{*}\right)$ critical points of index $k$. This implies that there must exist a Morse function $f$ with $c_{k}(f)$ critical points of index $k$, such that $c_{k}(f)=\beta_{k}\left(P^{*}\right)$. If not, then all Morse functions would have at least $\beta_{k}\left(P^{*}\right)+1$ critical points of index $k$, and this contradicts the maximality of the Poincaré polynomial $P^{*}$.

In fact we have a stronger result:

Theorem 3.3. The Morse inequalities hold for $P^{*}$.

Proof. We know that given any Morse function $f$ on $M$ the Morse inequalities hold for $f$. Also, $m_{f}(\lambda)=P^{*}+(1+\lambda) Q_{f}$. This implies that $c_{k}(f)=\beta_{k}\left(P^{*}\right)+c_{k}\left(Q_{f}\right)+$ $c_{k-1}\left(Q_{f}\right)$, for all $k$. The Morse inequalities for $f$ imply that

$$
\begin{gathered}
c_{k}(Q)+\beta_{k}\left(P^{*}\right)-\beta_{k-1}\left(P^{*}\right)+\ldots \pm \beta_{0}\left(P^{*}\right) \\
\geq \beta_{k}(M)-\beta_{k-1}(M)+\ldots \pm \beta_{0}(M) .
\end{gathered}
$$

Using (3) in Proposition 2.3, for all $k$ there exists $f \in \mathcal{F}(M)$ such that $c_{k}(f)=0$. Hence, we conclude that for all $k$ we have

$$
\beta_{k}\left(P^{*}\right)-\beta_{k-1}\left(P^{*}\right)+\ldots \pm \beta_{0}\left(P^{*}\right) \geq \beta_{k}(M)-\beta_{k-1}(M)+\ldots \pm \beta_{0}(M) .
$$

Using Theorem 3.1 and Theorem 3.3 the following theorem is immediate.

Theorem 3.4. Let $M$ be a simply connected orientable closed $n$-manifold with $n>$ 5. Then $P^{*}$ is realizable on $M$.

In dimensions two and three we have the following realizability theorem.

Theorem 3.5. Let $M$ be an orientable closed manifold of dimension two or three. Then $P^{*}$ is realizable on $M$. 
Proof. The case $n=2$ is trivial. In the case $n=3, P^{*}$ is realizable. This follows from results in $\mathrm{dR}$, where we use minimal Lyapunov graphs labelled with the Heegard genus of $M$ and handlebody theory to prove the existence and realizability of minimal Morse functions on $M$.

However, for $n$-manifolds $M, n=4,5$, or for non-simply connected manifolds of dimension $n>5$ we do not know whether or not $P^{*}$ is realizable.

Proposition 3.6. If $P^{*}$ is not realizable on $M$, then any Morse polynomial $P$ on $M$ has the property that if $\beta_{k}(P) \neq 0$ and $\beta_{k}\left(P^{*}\right)=0$ then $\beta_{k}(M)=0$.

Proof. If this were not true we would contradict the weak Morse inequalities for $P^{*}$ stated in Proposition 3.2

In the case of a two-dimensional manifold, it is easy to see that the maximal Poincaré polynomial is the Betti polynomial with coefficients in $\mathbf{Z}_{2}$ and it is always realizable.

In fact we have the following result:

Proposition 3.7. If $M$ is a connected 2-manifold, then $\mathcal{P}(M)$ contains a unique polynomial of degree 2 which is the maximal Poincaré polynomial.

Proof. By the connectedness of $M$, there are Morse functions $f$ with a unique maximum critical point and a unique minimum critical point, hence $c_{2}(P)=c_{0}(P)=1$. By Definition 2.1 we get:

$$
m_{f}(\lambda)=\lambda^{2}+c_{1}(P) \lambda+1+(1+\lambda) Q(\lambda),
$$

where $P$ is in $\mathcal{P}(M)$. Taking $\lambda=-1$, we obtain

$$
\chi(M)=2-c_{1}(P)
$$

and this formula determines $c_{1}(P)$ in a unique way.

By the above proposition, the set of Poincaré polynomials of connected 2-dimensional manifolds consists exactly of three polynomials, namely $P^{*}, P^{*}-\left(\lambda+\lambda^{2}\right)$ and $P^{*}-(1+\lambda)$; the first, the maximal one, is a Betti polynomial and is always realizable, the second is a Betti polynomial if the manifold is not orientable and the field coefficients are e.g. the reals, and the third never is a Betti polynomial.

The following table shows the maximal Poincaré polynomial of surfaces:

\begin{tabular}{|l|c|}
\hline$M$ & \multicolumn{1}{c|}{$P^{*}$} \\
\hline$S^{2}$ & $\lambda^{2}+1$ \\
\hline$T^{2}$ & $\lambda^{2}+2 \lambda+1$ \\
\hline$T^{2} \# T^{2}$ & $\lambda^{2}+4 \lambda+1$ \\
\hline$\underbrace{T^{2} \# \ldots \# T^{2}}_{n}$ & $\lambda^{2}+2 n \lambda+1$ \\
\hline$\underbrace{R P^{2}}_{n}$ & $\lambda^{2}+\lambda+1$ \\
\hline$R P^{2} \# R P^{2}$ & $\lambda^{2}+2 \lambda+1$ \\
\hline$\underbrace{R P^{2} \# \ldots \# R P^{2}}$ & $\lambda^{2}+n \lambda+1$ \\
\hline
\end{tabular}


For example, $m_{f}(\lambda)=5 \lambda^{2}+8 \lambda+3$ is realizable on $T^{2}$. In fact,

$$
m_{f}(\lambda)-P^{*}\left(T^{2}\right)=\left(5 \lambda^{2}+8 \lambda+3\right)-\left(\lambda^{2}+2 \lambda+1\right)=4 \lambda^{2}+6 \lambda+2 .
$$

Since $4 \lambda^{2}+6 \lambda+2=(1+\lambda) Q(\lambda)=(1+\lambda)(4 \lambda+2)$, these coefficients represent in some sense "extra" singularities that may be cancelled: 4 sources, 2 sinks and 6 saddles.

When representing Morse functions, using the Morse relations with Poincaré polynomials the remainder terms most often are bifurcating terms, i.e., terms that represent in some sense extra critical points or critical sets, that can either collapse, bifurcate or cancel among themselves.

However, if these Morse functions are in some sense minimal and we use Poincaré polynomials to write the Morse relations, then these remainder terms may in fact represent topological numerical invariants. Moreover, if the maximal Poincaré polynomial is used to write the Morse relations, then the coefficients of the maximal Poincaré polynomial, $\beta_{k}\left(P^{*}\right)$, are topological invariants of the manifold as we shall see subsequently.

\section{Coefficients of $P^{*}$ as topological invariants}

Recall that $P^{*}$ is the maximum Poincaré polynomial and its coefficients are $\beta_{k}\left(P^{*}\right)$. By definition we have that $\beta_{k}\left(P^{*}\right) \geq \beta_{k}(M, F)$, where $\beta_{k}(M, F)$ are the Betti numbers of the homology groups of $M$ with coefficients in a field $F$. The $\beta_{k}\left(P^{*}\right)$ are topological differential invariants of $M$; in fact, if $\beta_{k}\left(P^{*}\right)$ is the $k$-th coefficient of the maximum Poincaré polynomial, it is smaller than or equal to the number of critical points of Morse index $k$ of any Morse function $f$ on $M$. Actually, it could be defined as follows:

$$
\beta_{k}\left(P^{*}\right)=\min \left\{\#_{k}(f) \text { for } f \in C^{2}(M) \text { and } f \in \mathcal{F}(M)\right\},
$$

where $\#_{k}(f)$ is the number of critical points of $f$ of index $\mathrm{k}$.

Thus, if $F$ is a $C^{2}$ diffeomorphism, this number does not change. In fact, if $x$ is a critical point of index $k$ of $f$ on $M$, then $F(x)$ is a critical point of index $k$ of $f(F(\cdot))$ on the manifold $F(M)$. Some of these coefficients coincide with known topological invariants of $M$. The coefficients of the maximum Poincaré polynomial, $\beta_{k}\left(P^{*}\right)$, are topological invariants of the $n$-dimensional manifold $M$.

For example, for $n=2$ and $M$ orientable, $\beta_{1}\left(P^{*}\right)=$ twice the genus of $M$. If $n=2$ and $M$ is non-orientable, $\beta_{1}\left(P^{*}\right)=$ genus of $M$.

For higher dimensions we will define the Cornea genus which is a natural generalization of the definition of genus for the two-dimensional case. We define the Cornea genus of a smooth compact connected $n$-manifold $M, C(M)$, as the maximal number of mutually disjoint smooth compact connected two-sided codimension one submanifolds that do not disconnect $M$. This definition coincides with the classical definition of genus of a closed orientable two-manifold. In [Co] it is shown that for manifolds without boundary $C(M)=g\left(\pi_{1}(M)\right)$, where $g\left(\pi_{1}(M)\right)$ is the genus of the fundamental group of $M, \pi_{1}(M)$. The genus of a group $\pi, g(\pi)$, is the maximal rank $r$ of a free group $F_{r}$ such that there exists an epimorphism $\pi \rightarrow F_{r}$.

With this definition we can interpret the $\beta_{1}\left(P^{*}\right)$ coefficient of the maximum Poincaré polynomial, in many cases, as the genus of the $n$-dimensional manifold $M$. 
In general,

$$
\beta_{1}\left(P^{*}\right) \geq \beta_{1}(M) \geq C(M),
$$

the second inequality being a result in $[\mathrm{Co}]$.

For example, for $n=2$ and $M$ orientable, $\beta_{1}\left(P^{*}\right)=2 C(M)$. For a threedimensional closed connected orientable manifold $M$, results in $\mathrm{dR}$ imply that $\beta_{1}\left(P^{*}\right)=H(M)$, where $H(M)$ is the Heegard genus of $M$. Moreover, in Co it is shown that $C(M) \leq H(M)$ and equality holds if and only if $\pi_{1}(M)$ is free on $H(M)$ generators.

Results of Smale $\mathrm{Sm}$ assert that for a closed, smooth $n$-manifold $M$ which is $(m-1)$-connected, and $n \geq 2 m,(n, m) \neq(4,2)$, there exists a Morse function with the following number of critical points: $c_{0}=c_{n}=1$ and $c_{i}=0$ for $0<i<m$ and $n-m<i<n$. It then follows that for this manifold, $C(M)=0$. Hence for $n>4$, the existence of such a Morse function on $M$ implies that $\beta_{1}\left(P^{*}\right)=C(M)=0$. In fact, because of the existence of this function we can assert that $\beta_{i}\left(P^{*}\right)=0$ for $0<i<m$ and $n-m<i<n$.

Under the same hypothesis as above, Smale considers the special cases where the connectivity $m$ coincides with the mid-dimension of $M, n=2 m+1$ or $n=2 m$, and proves stronger results. In the case where $n=2 m+1, M=H \cup H^{\prime}, H \cap H^{\prime}=$ $\partial H=\partial H^{\prime}$ where $H, H^{\prime} \in \mathcal{H}(2 m+1, k, m)$. The set $\mathcal{H}(2 m+1, k, m)$ consists of handlebodies which are obtained by attaching $m$-disks, $k$ in number, to a $2 m+1$ disk and thickening them. In this case, because of the existence of such a function, we assert that: $\beta_{i}\left(P^{*}\right)=0$ for $i \neq m, m+1$ and $\beta_{m}\left(P^{*}\right)=\beta_{m+1}\left(P^{*}\right)=k$ where $k$ is the Heegard genus of $M$. In the case where $n=2 m, \beta_{i}\left(P^{*}\right)=\beta_{i}(M)$.

For simply connected $n$-manifolds, $n \geq 5$, we have no information on the coefficients of $P^{*}$. The only exception being homotopy spheres which by Smale's proof of the generalized Poincaré conjecture are homeomorphic to the sphere and hence admit a Morse function with one index $n$ and one index 0 critical point. Hence, $\beta_{i}\left(P^{*}\right)=0$ for all $i \neq 0, n$ and equals one otherwise. For non-simply connected $n$-manifolds we have the lower bound for $\beta_{1}\left(P^{*}\right)$ in (4).

\section{REFERENCES}

[Co] O. Cornea, The genus and the fundamental group of high-dimensional manifolds, Stud. Cerc. Mat. 41, n.3 (1989) pp.169-178. MR 90h:57022

[dR] K. de Rezende, Gradient-like flows on 3-manifolds, Ergod. Th. and Dynam. Sys. 13 (1993) pp.557-580. MR 94j:58146

[Fr] J. M. Franks, Homology and Dynamical Systems, CBMS 49 (1982). MR 84f:58067

[Sm] S. Smale, Generalized Poincaré's conjecture in dimensions greater than four, Annals of Mathematics 74 (1961) pp.391-406. MR 25:580

Departament of Applied Mathematics, University of Pisa, Pisa, Italy

E-mail address: benci@dm.unipi.it

Departamento de Matemática, Universidade Estadual de Campinas, 13083-970 CampINAS, SÃo PAUlo, Brazil

E-mail address: ketty@ime.unicamp.br 